\title{
782 PD-L1-TARGETED ISAC COMBINES MYELOID CELL ACTIVATION, IMMUNE-CHECKPOINT INHIBITION AND ADCP TO IMPROVE ANTI-TUMOR EFFICACY OVER ANTI- PD-L1 ANTIBODIES IN PRECLINICAL MODELS
}

Justin Kenkel, Rishali Gadkari, Karla Henning, Romas Kudirka, William Mallet, Po Ho, Ganapathy Sarma, Steven Chapin, Liz Bogaert, Jennifer Melrose, Matthew Zhou, Suprit Deol, Cindy Kreder, Yuyi Shen, Puneet Anand, Arthur Lee, Hai Li, Shelley Ackerman, Brian Safina, David Dornan, Michael Alonso, Marcin Kowanetz*. Bolt Biotherapeutics, Redwood City, CA, United States

Background PD-L1 is an immune checkpoint that regulates anti-tumor $\mathrm{T}$ cell responses and is expressed on tumor cells as well as tumor-infiltrating immune cells across many tumor types. Immune-stimulating antibody conjugates (ISACs) consist of tumor-targeting antibodies conjugated to immune stimulants and are designed to activate the innate and adaptive immune systems against tumor cells following systemic administration. Here we show that PD-L1-targeted TLR7/8 ISACs elicit robust myeloid cell activation which leads to improved anti-tumor responses compared to anti-PD-L1 treatment in pre-clinical tumor models.

Methods A panel of proprietary anti-PD-L1 antibodies was identified through a phage display screen and subsequently tested for PD-L1 binding affinity and specificity, PD-L1/PD-1 blocking, antibody-dependent cellular phagocytosis (ADCP) by myeloid cells, and anti-tumor efficacy. Lead antibodies were conjugated to proprietary TLR7/8 agonists, and the resulting PD-L1 ISACs were evaluated for in vitro myeloid cell activation and in vivo efficacy against syngeneic and xenograft tumors.

Results Anti-PD-L1 antibodies induced robust ADCP by myeloid effector cells and medium to strong PD-L1/PD-1 blockade in vitro. Selected antibodies inhibited the growth of syngeneic MC38-hPD-L1 tumors in vivo, confirming efficient immunecheckpoint blockade. The conjugated PD-L1 ISACs induced robust, target-dependent activation of myeloid cells when cocultured with PD-L1-expressing tumor cells, as measured by increased secretion of such cytokines as IL-12p70, IFN-alpha, and TNF-alpha. Importantly, myeloid activation was observed following co-culture with tumor cells having various levels of endogenous PD-L1 expression that was within the range of PD-L1 expression observed in human tumors. Systemically administered surrogate PD-L1 ISACs were well tolerated in mice and showed improved anti-tumor efficacy over anti-PDL1 antibodies, with significant tumor growth delay or complete responses frequently observed in syngeneic (e.g. MB49, MC38-hPD-L1) as well as xenograft (e.g. HCC1954-hPD-L1) tumor models.

Conclusions These data demonstrate the potential of a PD-L1targeted ISAC as a multifunctional therapeutic that may improve efficacy of PD-L1/PD-1 inhibition by combining three mechanisms of action into a single molecule: TLR-mediated myeloid cell activation, $\mathrm{T}$ cell activation through immunecheckpoint inhibition as well as ADCP.

Ethics Approval All animal studies were performed in accordance with Institutional Animal Care and Use Committee (IACUC)-approved protocols.

http://dx.doi.org/10.1136/jitc-2021-SITC2021.782 